\title{
Integrating verbal quantitative information in linear orderings
}

\author{
RICHARD A. GRIGGS and SANDRA L. SHEA \\ University of Florida, Gaine sville, Florida 32611
}

\begin{abstract}
Subjects learned and answered questions about four-term linear orderings described in paragraphs of text. Spacing along the dimension employed was varied by using verbal quantifiers ("just barely," "moderately," and "very much"). After learning the quantitative information, performance on ordinal information varied as a function of the quantitative difference between terms in the ordering. Reaction time was faster for large quantitative differences than for small quantitative differences. This result indicates that vague verbal quantitative information is integrated into the memory representation for an ordering and that such quantitative information does affect test performance.
\end{abstract}

Potts (1972) attempted to characterize the strategies college students employ when trying to remember linear orderings which have been described in paragraphs of text. In general, Potts had subjects study a paragraph which described a linear ordering along some dimension by using sentences such as "The wolf was smarter than the deer, the bear was smarter than the hawk, and the hawk was smarter than the wolf." Such an ordering can be represented symbolically as $\mathrm{A}>\mathrm{B}>\mathrm{C}>\mathrm{D}$, where $\mathrm{A}, \mathrm{B}, \mathrm{C}$, and $\mathrm{D}$ represent the terms of the ordering and " $>$ " represents some comparative adjective. The three presented pairs necessary to establish the ordering $(A>B, B>C, C>D)$ are referred to as adjacent pairs. The deducible nonadjacent relationships $(A>C$, B $>$ D, A $>$ D) are termed remote pairs. A true-false test followed the study period. The true sentences were the six adjacent and remote pairs; the false sentences were formed by reversing the terms in each of the true sentences (e.g., the false counterpart of A $>$ B would be $B>A$ ).

Potts (1972) found that performance (proportion correct or reaction time) on remote pairs was superior to performance on adjacent pairs. In fact, performance was a function of the distance separating the terms in the test statement; that is, the further apart the two terms, the better the performance. This suggests that subjects did not store the set or some subset of the pairs, but rather some more holistic representation of the ordering (cf. Bransford \& Franks, 1971). Potts (1972) offered two possible explanations, a rating scale model and an end-term anchoring model. Using a theoretical approach derived from the spatial imagery

The authors would like to thank Loren Cohen and Donald Keen for their assistance in conducting the experiment and analyzing the data. This research was supported by a research grant from the Social and Behavioral Sciences Council, University of Florida, to the senior author. Requests for reprints should be sent to Richard A. Griggs, Department of Psychology, University of Florida, Gainesville, Florida 32611. model proposed by Huttenlocher (1968) for three-term series problems, Potts developed a rating scale model which argues that subjects store a sequential arrangement of the terms along an imaginary spatial scale on the critical dimension. Spatial differences between the terms on the scale represent quantitative differences. The further apart two terms are on the scale, the easier they are to differentiate. Remote pairs would be placed further apart than would be adjacent pairs in a spatial representation. Thus, subjects would perform better on remote pairs, since they would be easier to differentiate.

The end-term anchoring model proposes that the first and last terms of the linear ordering serve as anchors for learning the other terms in the ordering. The ordering is learned from the end terms inward. The end terms of the ordering are learned first and remembered best, and performance on remote pairs is superior because those pairs are more likely to contain at least one end term ( $A$ or $D)$.

Potts (1972) was unable to discriminate between the models, since distance effects and end-term effects were confounded. Only one of the six adjacent and remote pairs did not contain an end term. Potts (1974a) investigated the rating scale model by manipulating the interval between the adjacent items in the ordering. Spacing along the dimension was varied by inserting verbal quantifiers ("just barely," "moderately," and "very much") into the presented adjacent pairs. No difference in proportion correct for small and large quantitative distances was found. Potts concluded that, since proportion correct on remote pairs was still superior to proportion correct on adjacent pairs, such superiority could not be accounted for solely in terms of the large quantitative differences separating the terms of the remote pairs, and that the findings, therefore, did not support the rating scale model.

Potts (1974a), however, never established that subjects learned the quantitative information. For example, in Experiment 2, subjects were instructed 
to remember both ordinal and quantitative information. For three groups, each employing a different coding strategy, the mean proportions correct on tests of ordinal information were high $(.903, .856$, and .825$)$, but very low for tests on the quantitative information (.556, .407, and .497). The means seem especially low since subjects were instructed to remember the quantitative information. The guessing probability on the latter tests was $1 / 3$. Thus, Potts failed to demonstrate that much of the information had actually been encoded and, hence, incorporated into the ordering. Therefore, if quantitative differences were not learned, it is difficult to agree with Potts' conclusion that they had no effect.

Potts (1974b) used six-term linear orderings $(A>B>C>D>E>F)$ to further test the two models. Such orderings provide an inner subordering $(B>C>D>E)$ not confounded by end-term effects. Thus, the effects of spatial distance independent of end-term effects could be examined. Study was selfpaced, so very few errors were made and, therefore, reaction time (RT) was used to study processing. When end-term effects were eliminated; RT was a simple decreasing function of inferential distance, thus supporting the rating scale model. To explain this, Potts (1974b) proposed a comparison process during retrieval whose completion time was presumed to be inversely related to inferential distance. Potts (1974b) does not specify the nature of the comparison process which leads to the inferential distance/reaction time effect. The rating scale model does seem a viable explanation, even though Potts' earlier study (1974a) failed to support it, since no effects of quantitative information were observed.

The effects of quantitative differences in linear orderings have been demonstrated, however, in other studies. Moyer (1973) found that the time required to determine which was the larger of two animals was an inverse function of the logarithm of estimated difference in animal size. This is similar to functions obtained when subjects make direct. perceptual comparisons of objects different in size. To explain this finding, Moyer proposed that subjects convert animal names to internal analog representations which preserve size. Names are compared by making "internal psychophysical judgments" about the representations. Larger differences between animals are represented as larger differences between the internal analogs. This results in increased discriminability and, thus, faster RTs.

Paivio (1975) has confirmed Moyer's (1973) findings for memory size comparisons within a particular conceptual category (animals) and extended them to comparisons across categories. Paivio's (1975) results have considerable generality, since they were obtained using size norms based on ratings by an independent group rather than by the experimental subjects.

The phenomenon that performance depends on distance between terms has been labeled the symbolic distance effect (SDE) by Moyer and Bayer (1976). According to Moyer and Bayer, the SDE is the finding that "the time needed to compare two symbols varies inversely with the distance between their referents on the judged dimension" (p. 320). Moyer and Bayer provided more evidence for the SDE by showing that the larger the absolute size difference between two objects (circles), the faster the comparison of their referent names (CVCs) when the ordinal distance between the named objects is held constant.

All the above experimental evidence for the effects of quantitative differences in linear orderings is for orderings not described in text. There is, however, some evidence that numerical values assigned to verbal quantifiers, such as those used by Potts (1974a), predict problem-solving behavior. Hersh and Carmazza (1975) demonstrated that subjects effectively integrate vague verbal quantitative information such as " $A$ is slightly smaller than B," and so forth. They found that numerical values assigned by subjects to the terms "slightly," "much," "smaller," and "larger" could be used a week later to predict solutions to linear syllogism problems such as " $A$ is slightly larger than $B, C$ is much smaller than B, therefore A is - units distant from C." Thus, Hersh and Carmazza (1975), contrary to Potts (1974a), demonstrated that subjects use this type of information in a problem-solving situation involving linear orderings.

The results of the ordering studies provide support for the effects of quantitative differences and the validity of a rating scale, analog type model for the comparison process in Potts' (1974b) dual-processing theory. If the quantitative information in a prose description of a linear ordering is learned, quantitative differences should affect the comparison process. The present study was designed to test this concept. A more optimal paradigm insured that the quantitative information was learned.

The procedure was similar to that employed by Potts (1974a), but with the following modifications. To insure that the quantitative information was incorporated into the subjects' representations of the ordering, subjects studied and were tested on the quantitative information twice before being tested on the ordinal information. Reaction time, an important additional measure of performance not measured by Potts (1974a), was recorded in order to possibly detect processing differences that might not be revealed in error rates (e.g., Potts, 1974b). It was expected that, if subjects learned the quantitative information and, thus, incorporated it into their representations of the linear orderings, this information would affect test performance. A control condition in which only ordinal questions were asked on the three repetitions was also employed. Since only ordinal information was tested, subjects did not have to learn the quantitative information and, therefore, incorporate it into their memory representations for the order. Results 
comparable to those of Potts (1974a) should be observed in this condition; that is, no effect of quantitative information on test performance.

\section{METHOD}

Subjects

Forty-six undergraduates at the University of Florida participated as part of introductory psychology course requirements. Six teen subjects were randomly assigned to the increasing quantitative differences condition and 16 to the decreasing quantitative differences condition. The other 14 subjects served in the control condition.

\section{Apparatus}

An Iconix four-field tachistoscope was used to present the stimuli and record reaction time. Three telegraph keys located near the subject's right hand were arranged in a small arc, with about $3 \mathrm{~cm}$ between each key. During a trial, the subject rested the forefinger of his right hand on the center key, and responded by moving that finger left or right to depress the appropriate key. Within each condition, the right key was true and false equally often.

\section{Materials}

The stimuli consisted of two paragraphs. Each of the two paragraphs described a linear ordering among a bear (A), hawk (B), wolf (C), and deer (D) on the dimension of intelligence. This ordering was used by Potts (1974a). Paragraph 1, the passage with increasing quantitative differences between terms, was the following: In a small forest just south of nowhere, $a$ deer, a bear, a wolf, and a hawk were battling for dominion over the land. It boiled down to a battle of wits, so intelligence was a crucial factor. The bear was just barely smarter than the hawk, the hawk was moderately smarter than the wolf, and the wolf was very much smarter than the deer. The battle was decided in this way and tranquility returned to the area. As in Potts (1974a), this ordering can be characterized as A.B.. C...D. Paragraph 2, the decreasing quantitative differences passage, was identical to Paragraph 1 except it described decreasing differences, A . . B . . C.D.

The three adjacent pairs were presented in each paragraph in a chained order $(A B, B C, C D)$. This order was employed since Smith and Foos (1975) found that subjects are more successful in constructing linear orderings if adjacent pairs are presented in a chained order. Potts (1974a) used a $C D, A B, B C$ presentation order which Smith and Foos found to be the least successful order for constructing an ordering.

A set of nine sentences was employed to test the subject's knowledge of the quantitative differences between the adjacent pairs in the ordering. For each adjacent pair, the ordered relation between the two terms in the pair was combined with each of the possible quantitative differences ("just barely," "moderately," and "very much"). For example, the three test sentences for the adjacent pair AB were "A was just barely smarter than B," "A was moderately smarter than B," and "A was very much smarter than B." For each paragraph, only three sentences with the appropriate verbal quantifiers were considered true.

A set of 12 test sentences was employed to test the subject's knowledge of the ordinal information. Six sentences were true (statements of the three adjacent pairs and the three remote pairs); six sentences were false. False sentences were formed by reversing the terms in each true statement.

All paragraphs and test sentences were typed on $15 \mathrm{~cm} \times 23 \mathrm{~cm}$ blank white cards, using an IBM Selectric typewriter and Orator typeface.

\section{Procedure}

A between-subjects design with three experimental conditions was employed. Paragraph 1 was used in the increasing quantitative differences condition and Paragraph 2 in the decreasing quantitative differences condition. Both paragraphs were used in the control condition.

Subjects were tested individually. After being given instructions about the experimental task, the subject was presented with a paragraph and had as much time as he wanted to study it. He tapped the right key to signal that he was ready to begin the test sequence. The first test consisted of the nine quantitative information questions, presented in a different random order for each subject. Subjects were instructed to answer as rapidly but as accurately as possible. Subjects made responses by pushing the appropriate key for each test sentence presented. Responses and reaction times were manually recorded.

On each test trial the following temporal sequence occurred: (1) The experimenter provided the verbal cue "Ready" and simultaneously activated the logic circuits controlling presentation; (2) $500 \mathrm{msec}$ after activation, an orientation fiald consisting of a "+" located at the same position as the first letter of the test item was shown; (3) after $1,000 \mathrm{msec}$ of the orientation field, the test item replaced the orientation display and the RT clock was started; and (4) the clock was stopped and the display terminated when the subject pressed a response key.

A second self-paced study period on the same paragraph and a second quantitative test sequence followed. After a third self-paced study period on the paragraph, subjects were tested on the ordinal information. For each subject, the randomization of the 12 test statements was different, with the restriction that no matching true and false sentences occurred in succession. For the ordinal information test, subjects were told that a sentence should be considered true if the information contained in it was either presented in the paragraph or was logically deducible from the information in the paragraph, and false if the information in it was not in the paragraph and could not be logically deduced from the information in the paragraph.

Subjects in the control condition were tested only on the ordinal test sentences presented in a different randomization for each of the three test sequences. Paragraphs 1 and 2 were employed equally often for the control subjects. Seven subjects studied and were tested on Paragraph 1, and seven subjects studied and were tested on Paragraph 2.

\section{RESULTS}

The data of the quantitative differences conditions and the control condition were analyzed separately. The results for the quantitative differences conditions will be presented first. The overall proportions correct on the three repetitions were $.77,1.0$, and .98 for the decreasing condition and $.85,1.0$, and .98 for the increasing condition. This definitely establishes that subjects knew the quantitative information by the third repetition.

A three-way ANOVA was performed on the ordinal RT data for the third repetition. The three factors were direction of quantitative difference (increasing vs decreasing), truth value (true vs false), and distance (adjacent vs remote). Only RTs for correct responses were included in this analysis and in all subsequent analyses to be described. The main effects of distance and direction were significant $[F(1,30)=5.30, p=.03$, and $F(1,30)=5.93, p=.02$, respectively]. No other effects were significant.

The mean RTs for adjacent and remote pairs for both increasing and decreasing quantitative differences are 
Table 1

Mean Reaction Times in Milliseconds for Quantitative Difference Conditions

\begin{tabular}{|c|c|c|c|c|}
\hline \multirow{2}{*}{ Direction } & \multicolumn{2}{|c|}{ Distance } & \multicolumn{2}{|c|}{ Size } \\
\hline & Adjacent & Remote & Small & Large \\
\hline $\begin{array}{l}\text { Increasing } \\
\text { Decreasing }\end{array}$ & $\begin{array}{ll}2,936 & (153) \\
2,435 & (104) \\
\end{array}$ & $\begin{array}{l}2,739 \quad(108) \\
2,160 \quad(77) \\
\end{array}$ & $\begin{array}{ll}2,988 & (161) \\
2,224 & (85) \\
\end{array}$ & $\begin{array}{ll}2,517 & (134) \\
2,174 & (93) \\
\end{array}$ \\
\hline
\end{tabular}

Note-Standard errors are given in parentheses.

given in Table 1. The distance effect is consistent with the results of Potts (1974a) and other previous linear ordering studies. Subjects were faster on remote pairs than on adjacent pairs. Reaction times in the increasing condition were consistently higher than those in the decreasing condition. No direction effect was observed in Potts (1974a); however, the quantitative information was not learned in that study.

The method employed by Potts (1974a) was used to classify pairs as large and small and yet not confound quantitative differences with distance. One adjacent pair and one remote pair represent small quantitative differences $(A B$ and $A C$ in $A . B . C \ldots D ; C D$ and $B D$ in A ...B ..C.D). One adjacent pair and one remote pair also represent large quantitative differences (CD and $B D$ in A.B.C..D; $A B$ and $A C$ in A ...B ..C.D). The choices make the test of the effect of quantitative differences orthogonal to the comparison between adjacent and remote pairs.

The mean RTs for pairs described by large and small quantitative differences for both the increasing and decreasing conditions are also presented in Table 1 . A three-way ANOVA was performed on the third repetition RT data for large and small pairs. Size of quantitative difference (large vs small), truth value, and direction were the factors. Both size and direction main effects were significant $[F(1,30)=5.04, p=.03$, and $F(1,30)=7.41, p=.01$, respectively]. The Direction by Truth Value interaction was also significant $[F(1,30)=10.61, p<.01]$. Thus, the effects of quantitative differences not observed by Potts (1974a) were clearly obtained in the present study.

The results were different in the control condition, in which subjects were tested three times on the ordinal information, but never on the quantitative information.
The mean RTs for pairs with large and small quantitative differences and for adjacent and remote pairs for all three repetitions are given in Table 2 . The means are provided for both increasing and decreasing quantitative differences since the direction factor turned out to be close to significant. Error rates were low, $4 \%$ or less for all three repetitions.

A four-way ANOVA was performed on the RT data for the three repetitions. The factors were direction, truth value, distance, and repetitions (1st, 2 nd, and 3 rd). The distance and repetitions main effects were significant $[F(1,12)=5.46, p=.03$, and $F(2,24)=17.47, \quad p<.001, \quad$ respectively. Reaction times to remote test statements were less than RTs to adjacent statements. As would be expected, mean RT decreased across repetitions. No other effects were significant, but the direction main effect was marginal $(p=.063)$.

To check for a size of quantitative difference effect, another four-way ANOVA was performed on the large and small RTs for the three repetitions. In this analysis, only the main effect for repetitions was significant $[F(2,24)=16.68, p<.001]$. The direction main effect was again marginal $(p=.058)$. No other effects approached significance. As predicted, no effect of quantitative differences was observed in the control condition, in which quantitative information did not have to be learned, but a distance effect was found.

\section{DISCUSSION}

The effects of quantitative information were as predicted. When the quantitative information was not required by the testing procedure, the performance on

Table 2

Mean Reaction Times in Milliseconds for All Three Repetitions in the Control Condition

\begin{tabular}{|c|c|c|c|c|c|}
\hline \multirow{3}{*}{$\frac{\text { Repetition }}{1}$} & \multirow{3}{*}{$\begin{array}{l}\text { Direction } \\
\text { Increasing } \\
\text { Decreasing }\end{array}$} & \multicolumn{2}{|c|}{ Distance } & \multicolumn{2}{|c|}{ Size } \\
\hline & & Adjacent & Remote & Small & Large \\
\hline & & $\begin{array}{ll}4,375 & (457) \\
3,420 & (228)\end{array}$ & $\begin{array}{ll}4,428 & (450) \\
2,737 & (164)\end{array}$ & $\begin{array}{ll}4,025 & (413) \\
2,960 & (223)\end{array}$ & $\begin{array}{ll}4,484 & (539) \\
2,791 & (165)\end{array}$ \\
\hline 2 & $\begin{array}{l}\text { Increasing } \\
\text { Decreasing }\end{array}$ & $\begin{array}{ll}3,986 & (332) \\
2,457 & (126)\end{array}$ & $\begin{array}{ll}3,553 & (266) \\
2,232 & (114)\end{array}$ & $\begin{array}{ll}3,762 & (352) \\
2,461 & (162)\end{array}$ & $\begin{array}{ll}3,391 & (262) \\
2,229 & (143)\end{array}$ \\
\hline 3 & $\begin{array}{l}\text { Increasing } \\
\text { Decreasing }\end{array}$ & $\begin{array}{ll}3,019 & (218) \\
2,142 & (137)\end{array}$ & $\begin{array}{rr}2,925 & (244) \\
1,901 & (91)\end{array}$ & $\begin{array}{ll}2,834 & (326) \\
2,044 & (107)\end{array}$ & $\begin{array}{ll}3,022 & (256) \\
1,988 & (105)\end{array}$ \\
\hline
\end{tabular}

Note-Standard errors are given in parentheses. 
the test pairs described by large and small quantitative differences was the same. The superiority of the large pairs was observed when learning of the quantitative differences was insured. This is what a rating scale model would predict.

The effect of direction of quantitative differences was not expected. It was significant in the quantitative differences conditions and close to significant in the control condition. In all conditions, the effect was the same: RTs for increasing quantitative differences were slower than those for decreasing quantitative differences. No definite explanation of this effect is proposed. A possible explanation may lie in the conflict between the direction of the differences between terms and the order of the terms on the dimension. As the quantitative difference increases, the position of the terms on the dimension decreases. In the decreasing quantitative differences condition, no such conflict exists. Further research is clearly needed to discover the origin of the direction effect. The critical main effects of distance and size in this study were significant and did not interact with direction.

The present results show that the mental representation for the linear ordering information preserves more than simply ordinal information about the terms compared. The data agree with those of Moyer (1973), Moyer and Bayer (1976), and Paivio (1975), in that they support an analog-like model for the representation of the ordering information. The present results indicate that the nature of the analog representation may be positions along an imaginal spatial dimension and that these function as analog devices which preserve quantitative information between terms.

The effects of quantitative differences observed in the present study present difficulties for some semantic coding models such as Potts' (1975) "split-in-half" model. According to the model, subjects learn an ordering in two distinct halves and can respond faster when the terms in a question are from different halves of the ordering than when they are not. Since a fourterm ordering was employed in the present study, for both large and small pairs, one pair spanned the two halves of the ordering and one did not. Thus, this semantic model would predict no difference in RTs for large and small quantitative differences; the difference, however, was clearly observed.

The semantic grouping model, however, can be modified to fit the present data. Following a suggestion by Potts (1975), if it is assumed that the order is not necessarily broken in half, but rather that the breaking place depends upon idiosyncratic characteristics of the paragraphs studied and/or individual subjects, such a modified model could account for the quantitative difference results. A semantic coding model with such a variable grouping assumption and the analog model make similar predictions. The two models, however, postulate different factors as the cause of the distance effect. The semantic coding model proposes that the time to compare two terms increases as the probability that the terms are grouped together increases. According to the analog model, comparison time will increase as the similarity between the magnitudes of the two terms increases.

Although the design of the present study does not allow one to distinguish between analog and semantic coding models, it is clearly established that the verbal quantitative information is integrated into the memory representation for a linear ordering described in meaningful text and affects subsequent test performance on the ordering information. Contrary to an earlier study by Potts (1974a), when learning of the quantitative information is insured, test performance is a function of the quantitative difference between terms in the ordering.

\section{REFERENCES}

Bransford, J. D. \& Franks, J. J. Abstraction of linguistic ideas. Cognitive Psychology, 1971, 2, 331-350.

Hersh, H. M. \& Caramazza, A. Integrating verbal quantitative information. Bulletin of the Psychonomic Society, 1975, 6, 589-591.

Huttenlocher, J. Constructing spatial images: A strategy in reasoning. Psychological Review, 1968, 75, 550-560.

MoYer, R. S. Comparing objects in memory: Evidence suggesting an internal psychophysics. Perception \& Psychophysics, 1973, 13, 180-184.

Moyer, R. S. \& BAYER, R. H. Mental comparison and the symbolic distance effect. Cognitive Psychology, 1976, 8, 228-246.

Paivio, A. Perceptual comparisons through the mind's eye. Memory \& Cognition, 1975, 3, 635-647.

Poтts, G. R. Information processing strategies used in the encoding of linear orderings. Journal of Verbal Learning and Verbal Behavior, 1972, 11, 727-740.

Ports, G. R. Incorporating quantitative information into a linear ordering. Memory \& Cognition, 1974, 2, 533-538. (a)

Potrs, G. R. Storing and retrieving information about ordered relationships. Journal of Experimental Psychology, 1974, 103, 431-439. (b)

Potrs, G. R. Bringing order to cognitive structures. In F. Restle, R. Shiffrin, J. Castellan, H. Lindman, \& D. Pisoni (Eds.), Cognitive theory [Vol. 1]. Hillsdale, N.J: Lawrence Erlbaum, 1975.

Smith, K. H. \& Foos, P. W. Effect of presentation order on the construction of linear orders. Memory \& Cognition, $1975,3,614-618$.

(Received for publication September 10, 1976; revision accepted November 26, 1976.) 